\title{
Force control in piezoelectric microactuators using self scheduled $H_{\infty}$ technique
}

\author{
Micky Rakotondrabe and Yann Le Gorrec \\ AS2M department, \\ FEMTO-ST Institute, \\ UMR CNRS 6174 - UFC / ENSMM / UTBM \\ 24, rue Alain Savary, Besançon 25000, FRANCE \\ corresponding author: mrakoton@femto-st.fr
}

\begin{abstract}
:
In micromanipulation and microassembly tasks, the manipulated micro-objects do not always have the same characteristics, such as compliance. Thus, both the static and dynamic models representing the force behavior respect to input sollicitations depend on the characteristics of of the manipulated micro-object. As a result, it is hard to synthesize a single controller able to ensure desired performances for all set of micro-objects, especially when their compliance range is very large. In this paper, we propose to model and control the manipulation force applied by piezoelectric microactuators by using a parameter dependent approach such that desired performances are ensured for all kind of manipulated objects. The resulting controller is said self-scheduled and easy to implement from numerical point of view.

First, we derive a model that is dependent on the characteristics of the manipulated micro-object. The strong hysteresis nonlinearity of the piezoelectric microactuator was compensated and the derived model is therefore linear. Afterwards, we design a self-scheduled controller using $H_{\infty}$ technique. In order to ensure the desired performances (micrometric accuracy, tens of millisecond of settling time) for any manipulated micro-objects, a parameter dependent controller is designed respect to the continuum of models. Finally, the efficiency of the proposed design procedure will be illustrated from experimental results.
\end{abstract}

Keywords: Parameter dependent, self-scheduled controller, $H_{\infty}$, force, piezoactuators, micromanipulation and microasembly

\section{INTRODUCTION}

Piezoelectric materials are very prized in the design of manipulators, actuators, sensors and robots for micro/nano manipulation and microassembly tasks. This is especially due to the high resolution and the high bandwidth that these materials can provide. One of their famous application is the piezoelectric microgripper (1)(2).

A microgripper is based on two piezoelectric cantilevers (piezocantilevers), generally with rectangular cross-section (Fig. 1). While one piezocantilever is used for the precise positioning, the second one can be used to control the manipulation force. In fact, the force control allows keeping the manipulated object inside the gripper. This also avoids the damage of some fragile micro-objects (optical microobjects, biological cells, etc...). Another possible application of the force control through the piezocantilever is the characterization and/or the treatment of biological objects.

In the litterature, many studies have been dedicated to the modeling and control of position, i.e. control of the deflection of the piezocantilever $(3)(4)(5)(6)(7)$. On the other hand, partial studies refer to the force control (8) (9) (10) and no continous and full works were performed. This was mainly due to the lack of convenient micro-force

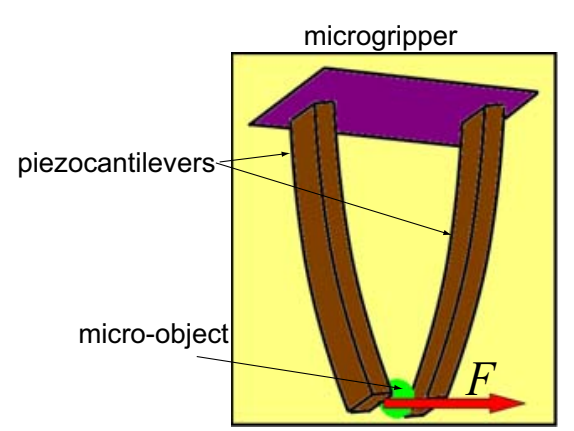

Fig. 1. A microgripper based on two piezocantilevers (piezoactuators).

sensors. Nevertheless, recent results on the measurement and the estimation of force in piezocantilevers $(11)(12)$ may be used to market the force control in these systems.

It is known that the model between the input control of a manipulator and the output manipulation force is dependent on the characteristics of the manipulated object, either using classical manipulators (13) or using piezoelectric manipulators (9). In micromanipulation/microassembly tasks, the manipulated micro-objects do not always have the same characteristics: the same microgripper can be used to manipulate biological cells, silicon based artificial 
objects and optical parts. In order to ensure specified performances for different manipulated objects, (9)(10) propose to use robust controllers. However, if the variation range of the characteristics of the different objects becomes important, the computed controller can not anymore ensure the performances, or even the stability. A selfscheduled controller should therefore be used.

In this paper, we propose to model the manipulation force in piezocantilevers using parameter dependent approach and to synthesize a self-scheduled $H_{\infty}$ controller. Behind the obtained performances, the advantage of the proposed technique is that the controller is simple from implementation point of view. The paper is organized as follows. Section-2 is dedicated to the modeling of the system. As the piezocantilever has a strong hysteresis nonlinearity, its compensation is briefly presented in the same section. In section-3, we present the synthesis of the controller and the experimental results.

\section{MODELING}

We consider one piezocantilever manipulating an object. The second piezocantilever of the microgripper is replaced by a rigid body. Using a lumped representation of the object, especially as a spring, we obtain the Fig. 2. We denote $\delta_{n c} \geq 0$ the distance between the manipulator and the object before the contact.

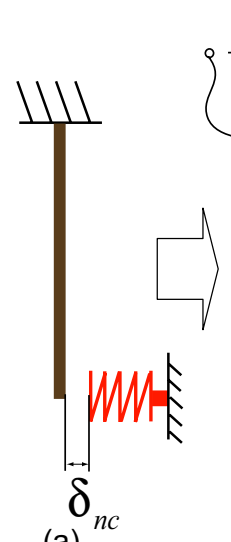

(a)

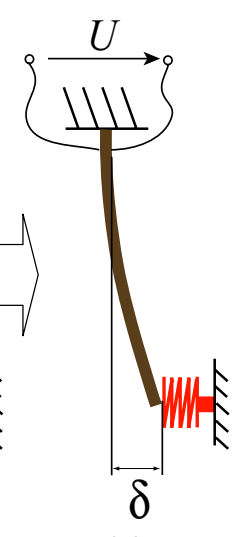

(b)
Fig. 2. A piezoactuator manipulating an object.

\subsection{Model of the piezoactuator}

The relation between the applied voltage $U$, the external force $F_{\text {ext }}$ applied at the piezocantilever's tip and the resulting deflection is as follow (see for eg. (6)):

$$
\delta=H_{s t}(U) \cdot D(s)+C_{r}(s) \cdot U+c_{p} \cdot D(s) \cdot F_{\text {ext }}
$$

where:

- $H_{s t}(U)$ represents the (static) hysteresis nonlinearity that characterizes the piezoelectric material,

- $D(s)$ is the normalized dynamic part, such as $D(0)=$ 1 ,

- $C_{r}(s)$ is a linear dynamic transfer that models the creep characteristic of the piezoelectric material,

- and $c_{p}$ is the elastic constant of the piezocantilever.
The model in (Eq. 1) is nonlinear. In order to further synthesize a linear controller, we decide to compensate the hysteresis $H_{s t}(U)$ while the creep will be considered as an external disturbance to be rejected. The principle of the hysteresis compensation is based on a precise modeling $H_{s t}$ and putting in cascade with the piezocantilever an inverse model $H_{s t}^{i n v}(\cdot)$. The output of the compensator is the voltage $U$ while its input is a deflection reference denoted $\delta_{r s}$. Applying this compensator in (Eq. 1), we obtain:

$\delta=H_{s t}\left(H_{s t}^{i n v}\left(\delta_{r s}\right)\right) \cdot D(s)+C_{r}(s) \cdot H_{s t}^{i n v}\left(\delta_{r s}\right)+c_{p} \cdot D(s) \cdot F_{e x t}$

which becomes:

$$
\delta=k \cdot D(s) \cdot \delta_{r s}+d_{c r}+s_{p} \cdot D(s) \cdot F_{\text {ext }}
$$

where $k \approx 1$ is the linear static gain, and $d_{c r}=d_{c r}\left(\delta_{r s}\right)=$ $C_{r}(s) \cdot H_{s t}^{i n v}\left(\delta_{r s}\right)$ is an input dependant disturbance that is linked to the creep.

To compensate the hysteresis $H_{s t}(\cdot)$ with $H_{s t}^{i n v}(\cdot)$, we propose to use the Prandtl-Ishlinskii (PI) approach. In this approach, the hysteresis model is based on the play operator, also called backlash operator (14). A play operator of unity slope is defined by:

$$
\delta^{e l}(t)=\max \left\{U(t)-r, \min \left\{U(t)+r, \delta^{e l}(t-T)\right\}\right\}
$$

where $T$ is the sampling time and $r$ the threshold of the operator.

Therefore, a hysteresis $H_{s t}$ is approximated by the sum of several play operators weighted by the gain (slope) $w_{i}$ (14). Let $n$ be the number of elements, so we have:

$$
\begin{aligned}
& \delta(t)=H_{s t}(U)= \\
& =\sum_{i=1}^{n} w_{i} \cdot \max \left\{U(t)-r_{i}, \min \left\{U(t)-r_{i}, \delta_{i}^{e l}(t-T)\right\}\right\}
\end{aligned}
$$

with $\delta$ being the output of the actuator and $\delta_{i}^{e l}$ being the output of the $i^{t h}$ play operator.

The PI hysteresis compensator $H_{s t}^{i n v}(\cdot)$ is also a PI-model, characterized by the thresholds $r_{i}^{\text {inv }}$ and the weightings $w_{i}^{i n v}$. These parameters can be analytically computed using the parameters of the direct model (15).

To identify and compute the direct model and the compensator of the hysteresis, we apply a sawtooth input voltage $U$ to the piezocantilever and the resulting deflection $\delta$ is measured. Fig. 3-a shows the setup: an optical sensor (Keyence LK-2520, with $\mathrm{nm}$ of resolution) is used to report the deflection. More details on the identification procedure and the computation of the compensator for piezocantilevers can are in our previous work (16). In Fig. 3-b, it is shown that the identified hysteresis model well fits to the experimental result. Fig. 3-c presents the experimental results when the hysteresis is compensated.

\subsection{Model of the manipulated object}

In order to further derive the model linking the new input control $\delta_{r s}$ and the output force, we need to model the object's deformation. Let $F=-F_{\text {ext }}$ be the manipulation force applied by the piezocantilever to the object. Let $c_{o}=\frac{1}{k_{o}}$ denote the compliance of the object, $k_{o}$ being its stiffness. Therefore, using Fig. 2, we have:

$$
\delta=c_{o} \cdot F+\delta_{n c}
$$



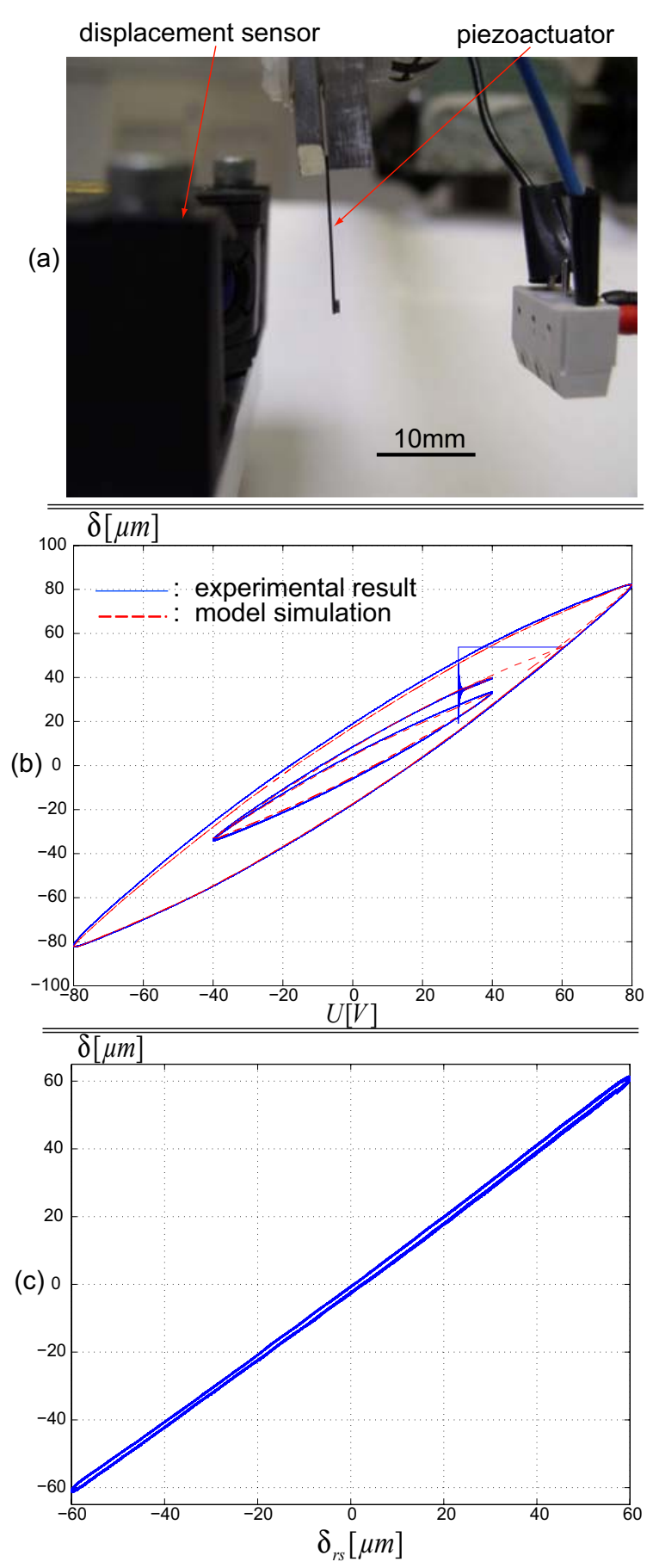

Fig. 3. (a) The experimental setup. (b) Hysteresis characteristic of the piezoactuator. (b) Piezoactuator's static characteristic after hysteresis compensation.

This object model is only static and does not account the effective mass and the viscous deformation. Such assumption is valid when the manipulated object is very small (micro-object) and when its stiffness is not too low.

\subsection{Model relating the output force and the input control}

Using the piezocantilever's linear model in (Eq. 3) and the object model in (Eq. 6), knowing that $F=-F_{\text {ext }}$, and re-arranging the computed expression at our convenience, we infer the model that links the input control $\delta_{r s}$ and the output force:

$$
F_{m}=\frac{1}{c_{o}}\left(\frac{k D(s)}{\left(1+\frac{c_{p}}{c_{o}}\right)} \delta_{r s}+\frac{1}{\left(1+\frac{c_{p}}{c_{o}}\right)} d\right)
$$

where $d$ is a disturbance and is defined by: $d=d_{c r}-\delta_{n c}$.

This model is parameter $c_{o}$ dependent. We propose to choose it as a scheduling parameter.

From the proposed expression in (Eq. 7), we provide the bloc diagram of the system to be controlled as depicted in Fig. 4. The objective is to separate the object parameter $c_{o}$ from an independant model (here, from $k \cdot D(s)$ ) as we can, and therefore to propose a parameter dependent controller scheme that is easy to implement.

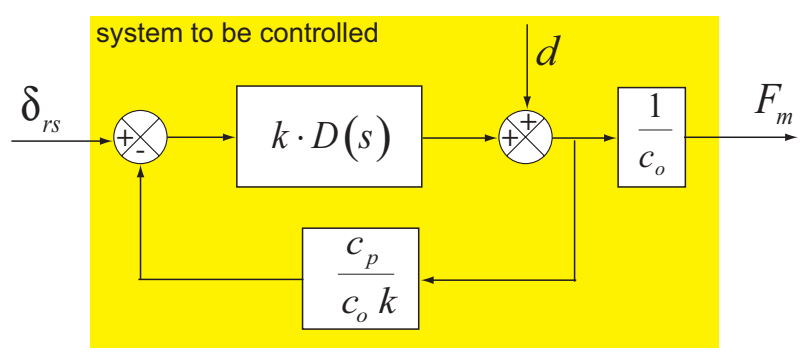

Fig. 4. Bloc diagram of the system to be controlled.

\subsection{Identification}

In the model of (Eq. 7), the elements to be identified are the static gain $k$, the compliance of the piezocantilever $c_{p}$ and the dynamic part $D(s)$. First, to identify $c_{p}$, we put a known mass at the tip of the piezocantilever. Using the resulting deflection, we derive: $c_{p}=2\left[\frac{\mu m}{m N}\right]$. Afterwards, we apply a step input $\delta_{r s}=60[\mu \mathrm{m}]$ to the system and the output $\delta$ is reported. Using the ARMAX method, we identify $k \cdot D(s)$. The experimental and the simulation results are plotted in Fig. 5-a and show that the model is enough accurate for a feedback controller synthesis. We have:

$$
k D(s)=\frac{0.06(s+7686)\left(s^{2}+8602 s+9.89 \times 10^{7}\right)}{(s+2950)\left(s^{2}+130 s+1.43 \times 10^{7}\right)}
$$

We notice that when observing the step response for a long period, the creep effect can be seen (Fig. 5-b). Here, an input $\delta_{r s}=60[\mu \mathrm{m}]$ generates a creep nearly $10[\mu \mathrm{m}]$ at the output. It correponds to $d_{c r}$ when using the maximal range of displacement.

\section{CONTROL}

The model developed as in (Eq. 7) is $c_{0}$-parameter dependent. According to the manipulated objects, the parameter's range may be very large and therefore a fixed controller, even robust, will not anymore ensure the performances, even so the stability. Therefore, we propose a scheduled controller in this section.

\subsection{Principle scheme of the self-scheduled control law}

To control the $c_{o}$ dependent model of the Fig. 4, we propose to use the $c_{o}$ dependent controller $\kappa\left(s, c_{o}\right)$ as presented in Fig. 6-a. The controller $\kappa$ also contains a fixed gain $C(s)$. After simplification, the bloc diagram of 


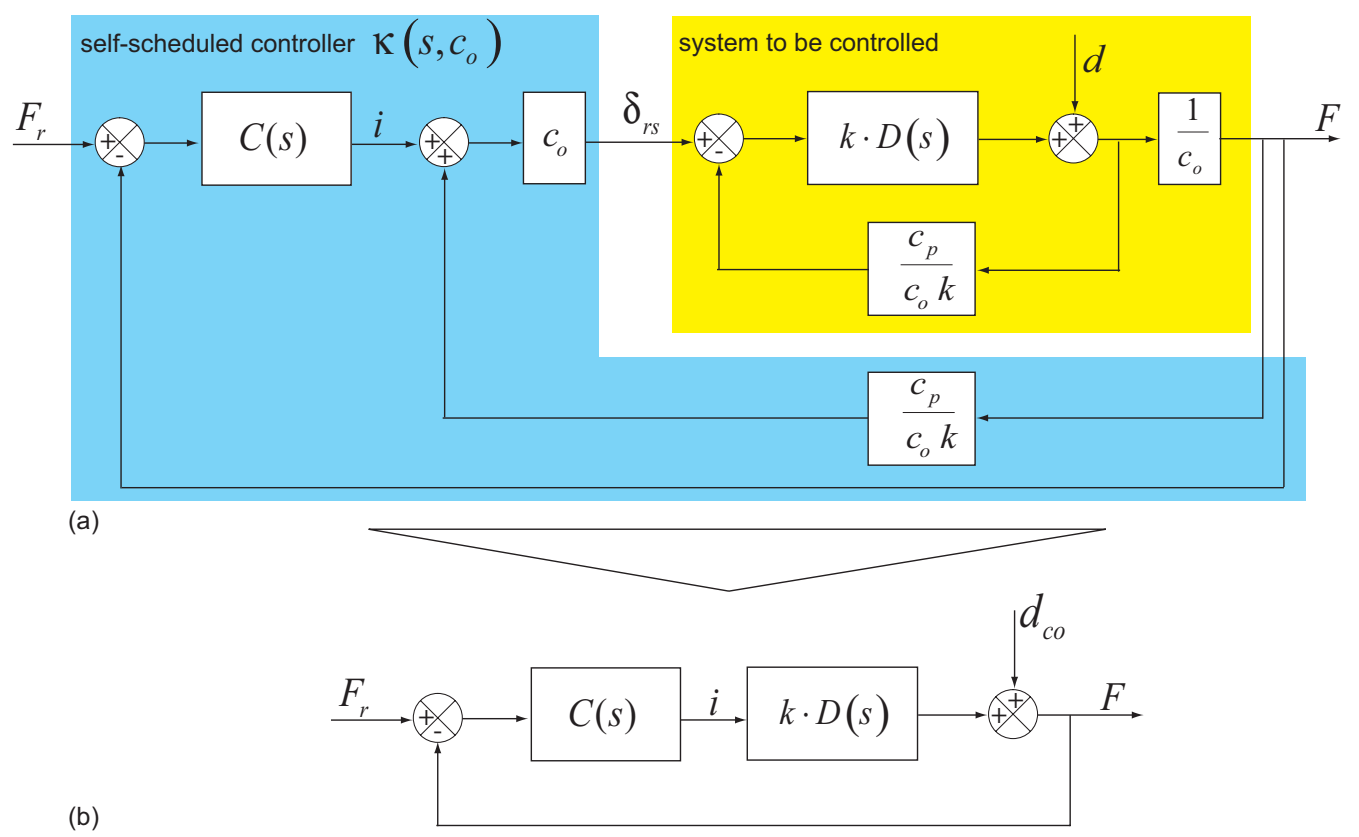

Fig. 6. (a) Bloc diagram of the closed-loop system. (b) Equivalent closed-loop scheme.
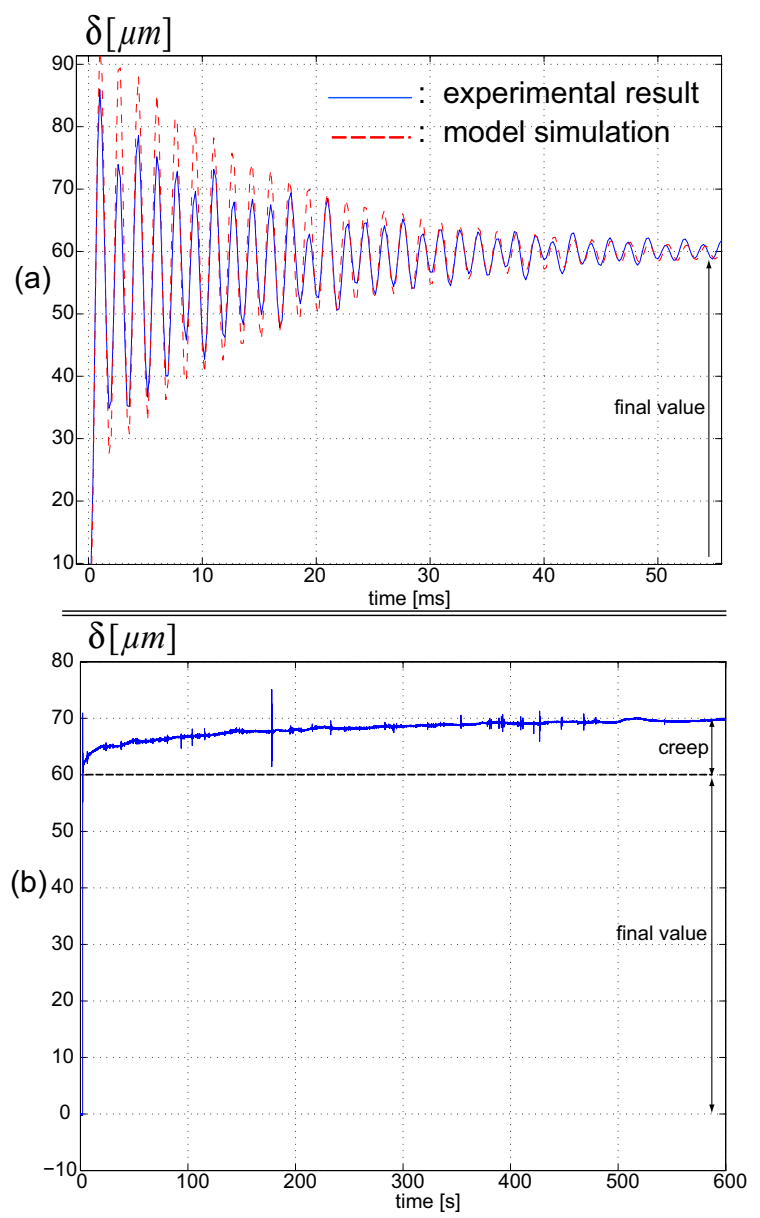

Fig. 5. (a) Step response of the piezoactuator (experimental result and simulation of $D(s)$ ). (b) Creep characteristic of the piezoactuator.

Fig. 6-a is equivalent to the bloc diagram presented in Fig. 6-b. Therefore, the problem comes back to find a fixed controller $C(s)$ using the fixed model $k D(s)$, which is more simple. Variable $i$ is the input of the new model and $d_{c o}=\frac{d}{c_{o}}$ is a disturbance to be rejected.

\section{2 $H_{\infty}$ controller design}

To compute $C(s)$, we use the $H_{\infty}$ standard technique in order to explicitely account the specifications. Notably, they concern 1) the tracking performances, 2) the disturbance $d_{c o}=\frac{d}{c_{o}}$ rejection, 3 ) and the limitation of the input control $i$.

Control design scheme Based on the above draft specifications, we derive the standard form (Fig. 7) where $W_{1}(s)$, $W_{2}(s)$ and $W_{3}(s)$ are the weighting functions for the tracking performances, the input signal limitation and the disturbance rejection respectively. The signals $o_{i}(i \in\{1,2\})$ are the output to be controlled while the reference input $F_{r}$ and signal $b$ concern the exogenous input signals. Using the figure, we have:

$$
\left\{\begin{array}{l}
o_{1}=W_{1} S F_{r}-W_{1} S W_{3} b \\
o_{2}=W_{2} S C F_{r}-W_{2} S C W_{3} b
\end{array}\right.
$$

where $S=\frac{1}{1+C k D}$ is the sensitivity. Applying the $H_{\infty}$ standard problem (17) to the previous equations, the problem comes back to find the controller $C(s)$ and an optimal value of $\gamma$ that satisfy the following constrains:

$$
\begin{gathered}
|S|<\frac{1}{\left|W_{1}\right|} \quad|S|<\frac{1}{\left|W_{1} W_{3}\right|} \\
|S C|<\frac{1}{\left|W_{2}\right|}|S C|<\frac{1}{\left|W_{2} W_{3}\right|}
\end{gathered}
$$

Choice of the weighting functions To define the weighting functions, the following detailed specifications are used.

For the tracking performances: the settling time needs to be lower than $30 \mathrm{~ms}$ and the statical error inferior to $1 \%$. In order to avoid a force overshot that may destroy the 
manipulated micro-objects, the overshot should be null. Therefore, we choose the following upperbond:

$$
\frac{1}{W_{1}}=0.01 \times \frac{(s+1)}{\left(\frac{0.03}{3} s+1\right)}
$$

For the input control signal limitation. In order to avoid high sollicitation to the actuator, we give an upperbound for the gain between the input control $i$ and the reference $F_{r}$ as follows:

$$
\frac{1}{W_{2}}=\frac{i}{F_{r}}=3.5
$$

Finally, for the disturbance rejection. Consider the disturbance equation $d=d_{c r}-\delta_{n c}$ (see (Eq. 7)). The disturbance is maximal when $\delta_{n c}=0$ and when the creep is obtained with the maximal range of use, i.e. $\delta_{c r}=10 \mu \mathrm{m}$ (see Fig. 5-b). Hence, an estimated maximal of $d_{c o}$ is: $d_{c o}=$ $\frac{d}{c_{o}}=\frac{10[\mu \mathrm{m}]}{0.5\left[\frac{\mu \mathrm{m}}{\mathrm{mN}}\right]}=20[\mathrm{mN}]$, where $0.5\left[\frac{\mu \mathrm{m}}{\mathrm{mN}}\right]$ is the considered minimal compliance in this paper but the user could choose another value. So, when the disturbance is maximal, we specify that its influence on the output is inferior to $1[\mathrm{mN}]$. Furthermore, we require that the maximal settling time of the disturbance rejection is $50 \mathrm{~ms}$. So, we choose:

$$
\frac{1}{W_{1} W_{3}}=\frac{1}{20} \times \frac{(s+1)}{\left(\frac{0.05}{3} s+1\right)}
$$

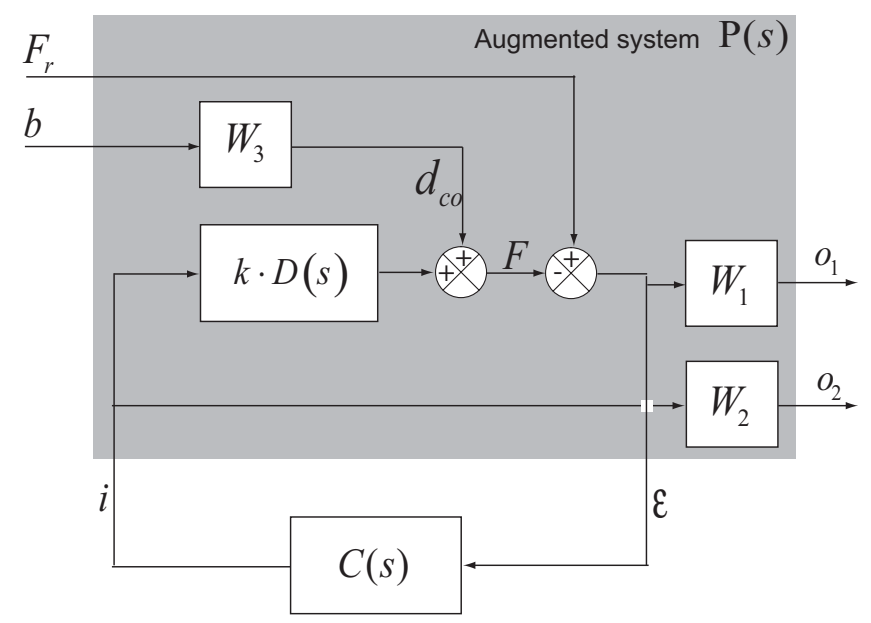

Fig. 7. Standard form.

Computation of the controller The controller is computed using the Glover-Doyle algorithm (18)(19) and the Matlab (c) software. The corrector $C(s)$ has an initial order of 6 . To reduce the time and memory consumption, we decide to reduce the order by using the balanced realization technique (20). Finally, we obtain a third order controller:

$$
C(s)=\frac{0.72(s+1236)\left(s^{2}+196 s+1.2 \times 10^{7}\right)}{(s+1)\left(s^{2}+1.1 \times 10^{4} s+1.01 \times 10^{8}\right)}
$$

\subsection{Experimental result}

The computed corrector $C(s)$ was introduced in the selfscheduled controller $\kappa\left(s, c_{o}\right)$ as in Fig. 6-a and the latter was implemented in the Matlab-Simulink and dSPACE real-time material. Instead of manipulating micro-objects, the experiments were performed with passive cantilevers.
Fig. 8-a and b picture the magnitudes of the sensitivity $S$ and of $C S$ respectively and of the different upper bounds.
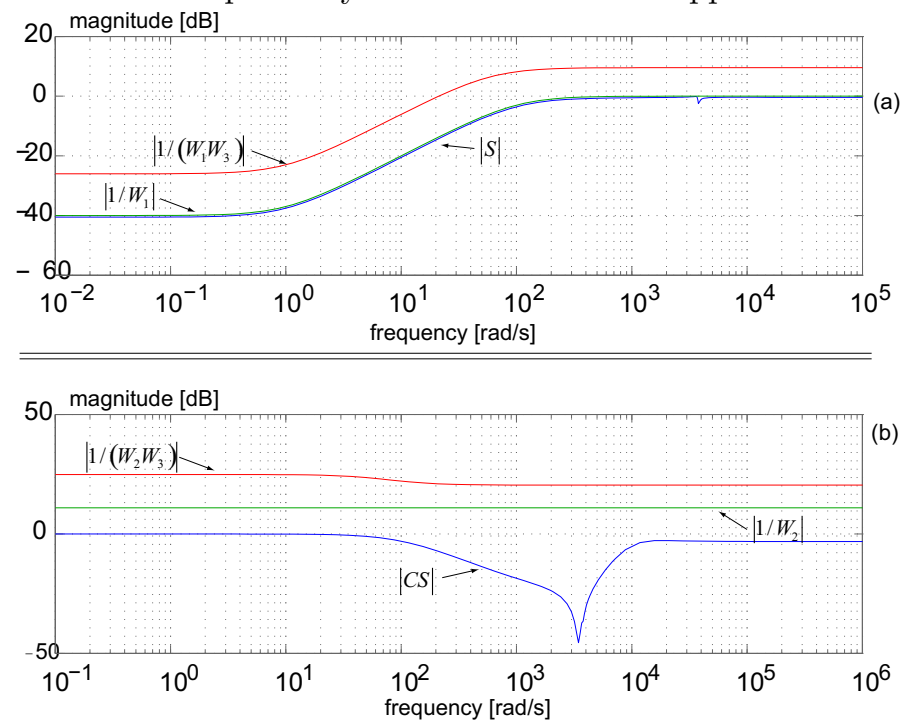

Fig. 8. Magnitudes of the transfers and of the upper bounds.

The used cantilevers have known compliance values. Two cantilevers were used: one flexible $\left(c_{o}=6.42\left[\frac{\mu m}{m N}\right]\right)$ and one rigid $\left(c_{o}=1.7\left[\frac{\mu m}{m N}\right]\right)$ (Fig. 9). For each manipulated

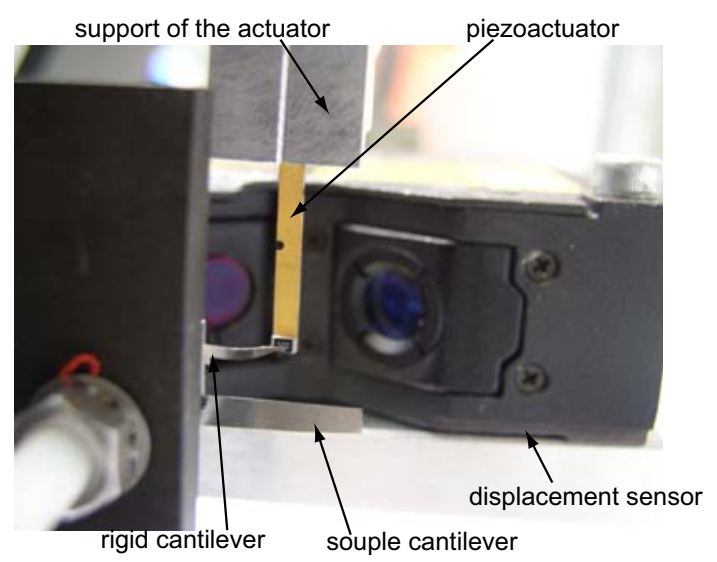

Fig. 9. A photography of the piezoactuator and the manipulated object.

passive cantilever, the corresponding compliance is manually introduced in the corrector $\kappa\left(s, c_{o}\right)$ and the latter is automatically scheduled. The force is measured using an estimation technique presented in previous work (10). Fig. 10 shows the experimental step response of the closedloop. It clearly shows that the specifications were satisfied, i.e. settling time, statical error and overshot, whatever the manipulated cantilever is (Fig. 10-a). The results indicates the efficiency of the proposed controller.

\section{CONCLUSION}

In this paper, we presented the modeling and the control of the force applied by a piezocantilever to a manipulated object. First, because of the strong nonlinearity of the piezoelectric material, we applied a hysteresis compensator based on the Prandtl-Ishlinskii approach. A linear model was afterwards developed. It has been shown that the 

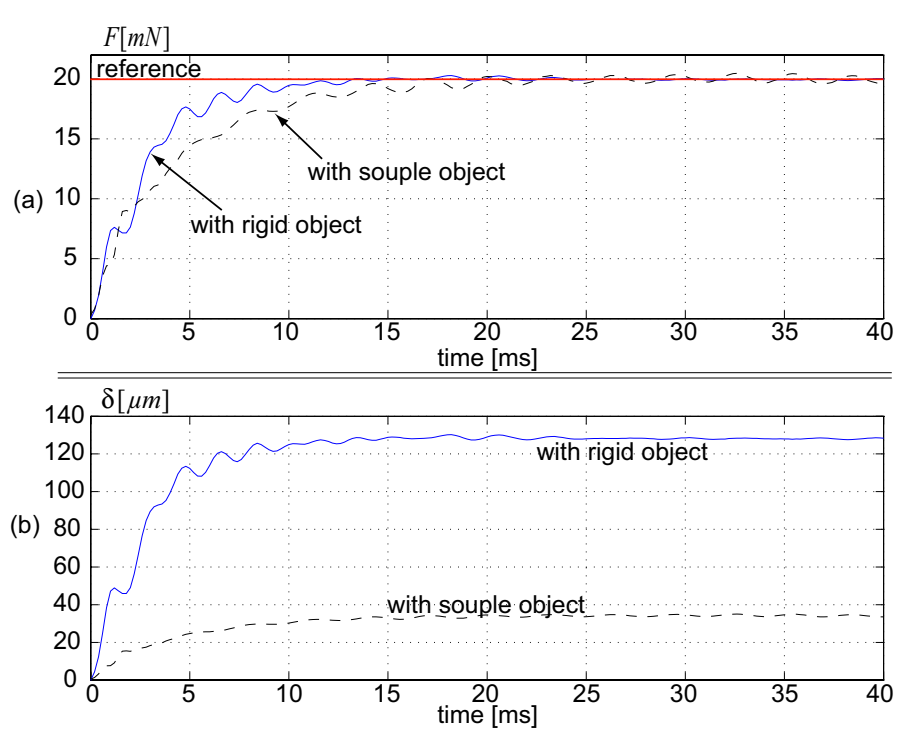

Fig. 10. Step response of the closed-loop system: (a) the force, (b) the deflection of the piezocantilever.

model is dependent on the characteristics of the manipulated object. In order to keep some specified performances for any kind of objects, we therefore proposed a selfscheduled $H_{\infty}$ control law. The controller is said selfscheduled since it is scheduled accordingly to the compliance of the manipulated object. The proposed scheme is easy from implementation point of view and the experimental results with two different objects demonstrate its efficiency.

\section{ACKNOWLEDGMENT}

This work is supported by the Conseil General du Doubs, France. The authors would like to thank David Guibert for his help on the mechanical design.

\section{REFERENCES}

[1] J. Agnus, J. M. Breguet, N. Chaillet, O. Cois, P. de Lit, A. Ferreira, P. Melchior, C. Pellet and J. Sabatier, "A smart microrobot on chip: design, identification and modeling", Proc. IEEE/ASME International Conference on Advanced Intelligent Mechatronics (AIM), Kobe - Japan, pp.685-690 2003.

[2] Xinhan Huang, Jianhua Cai, Min Wang, Xiadong Lv, "A piezoelectric bimorph micro-gripper with microforce sensing", IEEE International Conference on Information Acquisition (ICIA), July 2005.

[3] P. Ge and M. Jouaneh, "Tracking control of a piezoceramic actuator", IEEE Transactions on Control System Technology, 4, pp.209-216, 1996.

[4] B. M. Chen, T. H. Lee, C. C. Hang, Yi Guo and S. Weerasooriya, "An Hinfini almost disturbance decoupling robust controller design for a piezoelectric bimorph actuator with hysteresis", IEEE Transactions on control systems technology, vol.7, Nř2, Mar 1999.

[5] M. S. Tsai and J. S. Chen, "Robust tracking control of a piezoactuator using a new approximate hysteresis model", Transactions of the ASME Journal of Dynamic Systems, Measurement and Control, 125, pp.96-102, 2003.
[6] Micky Rakotondrabe, Yassine Haddab and Philippe Lutz, "Quadrilateral modelling and robust control of a nonlinear piezoelectric cantilever", IEEE - Transactions on Control Systems Technology (T-CST), Vol.17, Issue 3, pp:528-539, May 2009.

[7] S. Devasia, E. E. Eleftheriou, R. Moheimani, "A survey of control issues in nanopositioning", IEEE Transactions on Control Systems Technology, Vol.15, $N^{\circ} 15$, pp.802-823, 2007.

[8] T. Tanikawa and M. Kawai and N. Koyachi and T. Arai and T. Ide and S. Kaneko and R. Ohta and T. Hirose, "Force control system for autonomous micro manipulation", IEEE International Conference on Robotics and Automation (ICRA), pp.610-615, Seoul, Korea, 2001.

[9] Micky Rakotondrabe, Yassine Haddab and Philippe Lutz, "Modelling and $\mathrm{H}$-inf force control of a nonlinear piezoelectric cantilever", IEEE/RSJ International Conference on Intelligent Robots and Systems (IROS), pp.3131-3136, San Diego CA USA, Oct-Nov 2007.

[10] Micky Rakotondrabe, Cédric Clévy and Philippe Lutz, "Modelling and robust position/force control of a piezoelectric microgripper", IEEE International Conference on Automation Science and Engineering (CASE), pp:39-44, Scottsdale AZ USA, Sept 2007.

[11] Micky Rakotondrabe and Philippe Lutz, "Force estimation in a piezoelectric cantilever using the inversedynamics-based UIO technique", IEEE International Conference on Robotics and Automation (ICRA), pp:2205-2210, Kobe Japan, May 2009.

[12] Alexandru Ivan, Micky Rakotondrabe, Philippe Lutz and Nicolas Chaillet, "Current integration force and displacement self-sensing method for cantilevered piezoelectric actuators", Review of Scientific Instruments (RSI), Vol.80(12), 2126103, December 2009.

[13] S. D. Eppinger and W. P. Seering, "On dynamic models of robot force control", International Conference on Robotics and Automation, IEEE ICRA, April 1986.

[14] M. A. Krasnosel'skii and A. V. Pokrovskii, "Systems with hysteresis", Springer-Verlag, Berlin, 1989.

[15] K. Kuhnen and H. Janocha, "Inverse feedforwrad controller for complex hysteretic nonlinearities in smartmaterials systems", Control of Intelligent System, Vol.29, $N^{o} 3,2001$.

[16] M. Rakotondrabe, C. Clévy and P. Lutz, "Complete open loop control of hysteretic, creepd and oscillating piezoelectric cantilevers", IEEE Transactions on Automation Science and Engineering, DOI 10.1109/TASE.2009.2028617.

[17] G. J. Balas, J. C. Doyle, K. Glover, A. Packard and R. Smith, ' $\mu$-analysis and synthesis toolbox', The Matworks User's Guide-3, 2001.

[18] K. Glover and J. C. Doyle, 'State-space formulae for all stabilizing controllers that satisfy an $H_{\infty}$-norm bound and relations to risk sensivity', Systems \& Control Letters, vol.11, pp.167-172, 1988.

[19] J. C. Doyle, K. Glover, P. K. Khargonekar and B. A. Francis, 'State-space solutions to standard $H_{2}$ and $H_{\infty}$ control problems', IEEE Transactions on Automatic Control, AC 34 Nř8, pp.831-846, 1989.

[20] B. C. Moore, 'Principal component analysis in linear systems: controllability, observability and model reduction', IEEE Transactions on Automatic Control, AC26(1), 1981. 